\title{
CAPÍTULO 16: CARACTERIZAÇÃO DE Escherichia coli ISOLADAS DE SUABES DE FRANGO DE CORTE E DE Alphitobius diaperinus EM PERÍODO PRE ABATE
}

\section{CHAPTER 16. CHARACTERIZATION OF Escherichia coli ISOLATED FROM CHICKEN SWABS AND Alphitobius diaperinus IN PRE-SLAUGHTER PERIOD}

\author{
Maísa Fabiana Menck Costa ${ }^{1}$, Ana Angelita Sampaio Baptista ${ }^{2}$, Larissa Justino ${ }^{3}$; Beatriz Queiroz dos \\ Santos $^{4}$, Renata Katsuko Takayama Kobayashi ${ }^{5}$
}

\begin{abstract}
Resumo
A avicultura é uma potência econômica para o Brasil. A utilização de antibióticos na produção animal pode levar a uma maior pressão de seleção sobre os microrganismos e consequentemente selecionar micro-organismos resistentes. Escherichia coli é apontada como um importante bioindicador de resistência aos antimicrobianos. O objetivo deste estudo foi determinar o perfil fenotípico de resistência aos antimicrobianos de Escherichia coli presentes na microbiota intestinal de aves no final do ciclo produtivo. Foram colhidos suabes de cloaca de frangos de corte (60 aves) e Alphitobios diaperinus. As amostras foram semeadas em Ágar MacConkey suplementado ou não com ciprofloxacina, e de cada meio foi selecionada uma amostra de Escherichia coli. O teste de sensibilidade aos antimicrobianos e a detecção fenotípica de betalactamases de espectro estendido (ESBL) foram realizados de acordo com CLSI e BrCast. Foram analisados 20 isolados de E. coli de suabes cloacais e quatro de Alphitobious diaperinus, os quais apresentaram elevada resistência a Sulfametoxazol + trimetoprim e ampicilina, e 100\% dos isolados em Ágar MacConkey suplementado com ciprofloxacina foram multirresistentes. É possível concluir que existe um alto perfil de resistência em isolados de Escherichia coli da microbiota intestinal de frangos de corte no período pré-abate e de Alphitobius diaperinus e que este pode atuar como veiculador e manter estes micro-organismos no ambiente.
\end{abstract}

Palavras-Chaves: Escherichia coli, Frango de corte, Resistência antimicrobianos, Ciprofloxacina.

\begin{abstract}
Poultry farming is an economic power for Brazil. The use of antibiotics in animal production can lead to greater selection pressure on microorganisms and consequently select resistant microorganisms. Escherichia coli is identified as an important bioindicator of resistance to antimicrobials. The aim of this study was to determine the phenotypic resistance profile to Escherichia coli antimicrobials present in the intestinal microbiota of birds at the end of the production cycle. Swabs from broilers (60 birds) and Alphitobios diaperinus were collected. The samples were seeded on MacConkey agar supplemented or not with ciprofloxacin, and a sample of Escherichia coli was selected from each medium. The sensitivity test to antimicrobials and the phenotypic detection of extended-spectrum beta-lactamases (ESBL) were performed according to CLSI and BrCast. Twenty $E$. coli isolates from cloacal swabs and four Alphitobious diaperinus were analyzed, which showed high resistance to Sulfametoxazol + trimethoprim and ampicillin, and 100\% of the isolates on MacConkey Agar supplemented with ciprofloxacin were multidrug-resistant. It is possible to conclude that there is a high

\footnotetext{
${ }^{1}$ Mestranda em Microbiologia, CCB/UEL, maisafabi@ hotmail.com

${ }^{2}$ Docente em Medicina aviária, CCA/HV/UEL, anaangelita@uel.br

${ }^{3}$ Mestranda em Medicina Veterinária, FMVZ/UNESP, larissajustino7@ hotmail.com

${ }^{4}$ Pós graduanda em Medicina aviária, DMVP/UEL, beatriz.queiroz@uel.br

${ }^{5}$ Pós Doutorado pelo Institut Armand-Frappier, Professor Associado UEL, kobayashirkt@uel.br
} 
resistance profile in isolates of Escherichia coli from the intestinal microbiota of broilers in the pre-slaughter period and of Alphitobius diaperinus and that it can act as a carrier and maintain these microorganisms in the environments.

Keywords: Escherichia coli, Broiler, Antimicrobial resistance, Ciprofloxacin.

\section{Introdução}

O Brasil está entre os três maiores produtores de carne de frango do mundo, com a produção de aproximadamente 13 mil toneladas, atualmente é o principal país exportador, sendo o Paraná o estado brasileiro que lidera a produção e exportação (ABPA, 2019; IBGE, 2017).

O consumo per capita brasileiro da carne de frango é $42 \mathrm{~kg} / \mathrm{ano}$ (ABPA, 2018; OECD/FAO, 2019), sendo uma das proteínas de maior consumo, se enquadra como um veículo de alto risco na disseminação de patógenos zoonóticos e reservatório de resistência a antibióticos (OECD/FAO, 2019; NEU, 1992; WITTE, 1998). Uma vez que um ambiente apresente resistência, esta pode chegar a outros locais e outros países (CDC, 2019).

Em 2006 a União Europeia proibiu o uso de antimicrobianos como promotores de crescimento, desde então medidas alternativas vem sendo buscadas, o frango de corte demanda da utilização de antimicrobianos de três formas, como promotores de crescimento, como preventivos e como terapêutico. Porem independentemente da forma, e da espécie que utilize, a utilização de antimicrobianos leva a uma maior pressão de seleção e consequentemente a um maior número de microrganismos resistentes (CYOIA et al., 2019; THANNER; DRISSNER; WALSH, 2016; VAN IMMERSEEL et al., 2016).

A resistência a antimicrobianos é definida como a capacidade que um organismo adquire de poder sobreviver a um fármaco com ação bactericida ou bacteriostática. Pode ocorrer pela pressão de seleção, ou seja, os microrganismos sensíveis morrem e os organismos resistentes sobrevivem podendo aumentar sua população, por mutações pontuais no cromossomo, ou por disseminação de genes que conferem resistência (CDC, 2019).

A disseminação de genes por meio da transferência de material genético pode ocorrer por conjugação, por transdução ou por transformação. A conjugação é definida pela habilidade que alguns microrganismos possuem de transferência de material genético (plasmídeos) por pili sexuais, a transdução ocorre com o auxílio de bacteriófagos e a transformação ocorre pela absorção de DNA presente no ambiente (GIGUÈRE et al., 2010).

Escherichia coli pode ser considerada um bioindicador de resistência aos antimicrobianos, uma vez que existem muitos estudos desse microrganismo e uma importante correlação desta espécie microbiana entre humanos e animais (SEO, SHIM e LEE, 2019; 
OIKARAINEN et al., 2019; PESCIAROLI et al., 2020; THEOBALD et al., 2019; JOHNSON et al., 2012; PITOUT, 2012; MELLATA, 2013).

Escherichia coli é um bacilo anaeróbico facultativo, Gram negativo não formador de esporos, que pode apresentar flagelos peritríquios, pertence a ordem Enterobacteriales e família Enterobacteriaceae (FU et al., 2013; WILLIAMS et al., 2010).

Trata-se de um microrganismo que pode ser comensal e estar presente no trato gastrintestinal da grande maioria dos animais, dentre esses os humanos e as aves, porém também pode apresentar-se como um microrganismo patogênico, levando a enfermidades no trato gastrointestinal de humanos (ex.: O157:H7), ou no sistema urinário (ex.: J96) e em aves, levar a um quadro de colibacilose (BIDÊ; BANARCORSI; BINGEN, 2012; KAPER; KATARO; MOBLEY, 2004).

Os Alphitobius diaperinus (Cascudinhos) são insetos encontrados em aviários, e colaboram com a permanência de microrganismos patogênicos ou comensais em galpões de frangos de corte e pela permanência de microrganismos resistentes no ambiente avícola, quando não for realizado um processo de fermentação e eliminação dos insetos adequadamente (DAVIES; WRAY, 1995; CHERNAKI-LEFFER et al., 2002; VITTORI et al., 2007).

A resistência a antimicrobianos é um relevante tema na atualidade, de acordo com O’Neil (2016) estima-se que em 2050 haverá aproximadamente 10 milhões de mortes ano devido à resistência aos antimicrobianos. Mediante a situação atual com uma pandemia e altas taxas de mortalidade, fatores que afetam a vida são e serão importantes pontos de atenção e cuidado (WHO, 2020). Nos EUA os dados apontam que em 2018 ao menos 2,8 milhões de pessoas foram infectadas por microrganismos resistentes e mais de 35 mil mortes/ ano (CDC, 2019).

Dentre os antimicrobianos utilizados tanto na medicina humana quanto na medicina veterinária está a ciprofloxacina. Trata-se de uma quinolona, com espectro de ação contra a maioria dos bacilos Gram negativos, que em humanos pode ser utilizada para o tratamento contra infecções causadas por Escherichia coli, Proteus mirabilis e Pseudomonas aeruginosa (KUPCZIK et al., 2009).

A presença de microrganismo na microbiota de aves no período pré abate, é um dado importante pois pode representar a contaminação presente nas linhas de abate, com conteúdo intestinal (MELLATA, 2013).

O objetivo do presente estudo foi determinar o perfil de resistência fenotípico de Escherichia coli isoladas de frango de corte e de Alphitobius diaperinus no final do ciclo produtivo. 


\section{Material e Métodos}

\section{Colheita de Material}

As colheitas foram realizadas em duas granjas de frango de corte do norte do Paraná, sempre respeitando um período máximo de três dias anteriores ao abate. Em cada granja foram coletados de forma aleatória suabes cloacais de 30 aves. Estes foram armazenados em meio de transporte Cary-Blair e mantidos refrigerados até serem encaminhados ao Laboratório de Medicina Aviária para processamento.

Também foram coletados aleatoriamente ao longo de toda a extensão de cada galpão aproximadamente 50 Alphitobius diaperinus em potes de coleta estéreis e mantidos refrigerados até o processamento.

\section{Isolamento de Escherichia coli}

Os suabes (30) de cada granja foram agrupados em cinco pools, e incubados em água peptonada tamponada (Accumedia), por $18-20$ horas a $37^{\circ} \mathrm{C}$. Os cascudinhos foram colocados em solução salina estéril por 10 minutos, na proporção de 1:10, seguidos de imersão em álcool $70^{\circ} \mathrm{C}$ por $10 \mathrm{~min}$, retirado o excesso de umidade em papéis estéreis e então macerados em cadinhos com o auxílio de pistilos, ambos estéreis e incubados em água peptonada tamponada (Accumedia), na proporção de 1:10, por $18-20$ horas a $37^{\circ} \mathrm{C}$.

Posterior as amostras foram plaqueados em ágar MacConkey (Acumedia) sem adição de antimicrobianos (MC1) e em ágar MacConkey suplementado com ciprofloxacina $8 \mu \mathrm{g} / \mathrm{mL}$ (MC 2) (CLSI, 2019). De cada placa foi selecionada uma colônia que apresentava morfologia característica da espécie, que foram submetidas a seis testes de triagem bioquímica Ágar tríplice açúcar (TSI), Ágar indol sulfeto motilidade (SIM), Citrato, Urease, Celobiose, Lisina e Sorbitol, os dois isolados foram armazenados a $-20^{\circ} \mathrm{C}$ até o momento do uso, Tabela 1.

\section{Identificação de Escherichia coli}

Os isolados lactose positiva em ágar MC 1 e $\mathrm{MC}$ 2, foram submetidos a triagem bioquímica e identificados como E. coli quando: Ágar tríplice açúcar (TSI) - com base e bisel ácidos, produção negativa para Sulfeto de Hidrogênio (H2S) e resultado variável para a

produção de gás; Ágar indol sulfeto motilidade (SIM) - com produção de indol positiva, motilidade variável e negativo para a produção de H2S; Citrato, Urease e Celobiose - negativos assim como Lisina e Sorbitol- positivos. 


\section{Teste fenotípico de resistência a antimicrobianos}

O teste fenotípico de resistência foi realizado por aproximação de discos, conforme descrito pelo CLSI (2019) e BrCast (2019). Os antimicrobianos utilizados foram: Amoxacilinaácido Clavulânico (AMC - $20 / 10 \mu \mathrm{g}$ ), Aztreonam (ATM - 30 $\mu \mathrm{g}$ ), Cefotaxima (CTX - $30 \mu \mathrm{g}$ ),

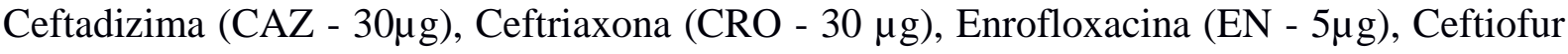

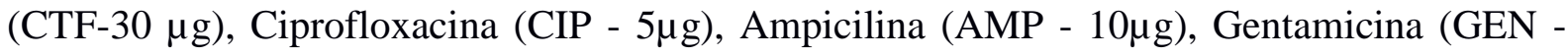

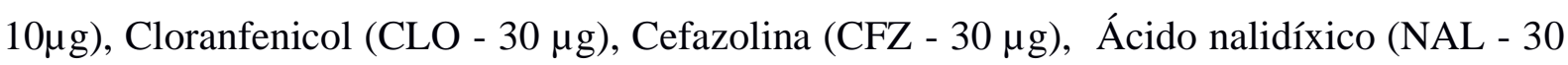

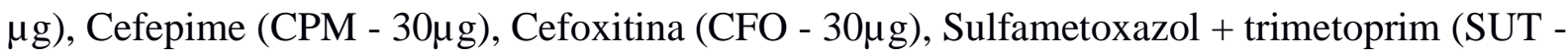
1,25/23,75 $\mu \mathrm{g}$ ), Imipenem (IMP - $10 \mu \mathrm{g}$ ), Tetraciclina (TET - $30 \mu \mathrm{g}$ ), Fosfomicina (FOS - 200 $\mu \mathrm{g})$. A cepa de Escherichia coli ATCC 25922 foi utilizada como controle padrão para o teste de antibiograma. Foram considerados isolados multirresistentes a drogas (MDR), os que apresentaram resistência a três ou mais classes de antimicrobianos (MAGIORAKOS et al., 2012).

Tabela 1. Apresentação do número de isolados com relação a sua origem.

\begin{tabular}{|c|c|c|c|}
\hline \multicolumn{2}{|c|}{ Meio de Cultura/ Origem Isolado } & $\begin{array}{c}\text { Ágar MacConkey } \\
\text { (Número de isolados) }\end{array}$ & $\begin{array}{l}\text { Ágar MacConkey CP } \\
\text { (Número de isolados) }\end{array}$ \\
\hline \multirow{6}{*}{ 莺 } & Suabe 1 & 1 & 1 \\
\hline & Suabe 2 & 1 & 1 \\
\hline & Suabe 3 & 1 & 1 \\
\hline & Suabe 4 & 1 & 1 \\
\hline & Suabe 5 & 1 & 1 \\
\hline & Alphitobius diaperinus & 1 & 1 \\
\hline \multirow{7}{*}{ 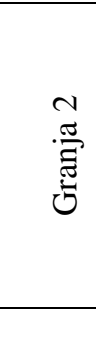 } & Suabe 1 & 1 & 1 \\
\hline & Suabe 2 & 1 & 1 \\
\hline & Suabe 3 & 1 & 1 \\
\hline & Suabe 4 & 1 & 1 \\
\hline & Suabe 5 & 1 & 1 \\
\hline & Alphitobius diaperinus & 1 & 1 \\
\hline & Total & 12 & 12 \\
\hline
\end{tabular}

*Ágar MacConkey 1 = sem suplementação com antimicrobianos; Ágar MacConkey 2= MacConkey suplementado com ciprofloxacina.

Fonte: Própria (2020).

\section{Teste fenotípico da produção de $\beta$-lactamase de espectro expandido}

A avaliação da produção de $\beta$-lactamase de espectro expandido (ESBL), foi realizada de acordo com o descrito pelo CLSI (2018) e BrCast (2017), com 20 mm de distância halo halo, entre os discos de amoxacilina- ácido clavulânico (AMC - 20 / $10 \mu \mathrm{g}$ ), um monobactâmco aztreonam (ATM - 30 $\mu \mathrm{g}$ ) e as cefalosporinas cefotaxima (CTX - $30 \mu \mathrm{g}$ ), ceftadizima (CAZ $30 \mu \mathrm{g})$ e ceftriaxona (CRO - $30 \mu \mathrm{g}$ ). A formação de um halo fantasma, ou distorção dos halos 
de cefalosporina ou monobactâmico quando em contato com a difusão do ácido clavulânico no meio de cultura demonstram a produção de ESBL.

\section{Resultados e Discussão}

Foram isolados ao total 24 Escherichia coli, sendo destas 12 a partir do Ágar MacConckey suplementado com ciprofloxacina e 12 sem a suplementação de antimicrobiano no meio de cultura, sendo 20 isolados de suabes cloacais e quatro isolados de Alphitobius diaperinus.

Tanto em MC1 quanto em MC2 foi observado crescimento microbiano e isolamento de Escherichia coli. Yassin et al. (2017) observaram que mais de 55\% dos seus isolados de origem avícola apresentaram resistência a ciprofloxacina sem necessidade de pré-seleção com antimicrobianos. Giufrè et al. (2012) utilizaram 101 cepas de Escherichia coli, destas 33 resistentes a ciprofloxacina, com o objetivo de comparar isolados de humanos e isolados de aves com relação ao genótipo, se eram compartilhados ou não. Em ambos os trabalhos observaram uma alta prevalência de resistência a ciprofloxacina, porém nenhum utilizou uma seleção prévia.

\section{Teste fenotípico de resistência a antimicrobianos}

Nos 10 isolados de suabe cloacal de ágar MacConkey sem suplementação com ciprofloxacina foi possível observar que o 50\% (5/10) apresentaram perfil de resistência ampicilina, seguidos para ciprofloxacina, ácido nalidíxico e sulfametoxazol + trimetoprim com $20 \%(2 / 10)$ e fosfomicina, cefazolina, gentamicina, enrofloxacina e cefotaxima com $10 \%(1 / 10)$ de resistência, Figura 1.

Dos 10 isolados de suabe cloacal proveniente do ágar MacConkey (8ug Cipro) observou-se $100 \%$ (10/10) de resistência ao ácido nalidíxico, pois trata-se da mesma classe antimicrobiana do agente de pré seleção, também apresentou 100\% (10/10) de resistência a enrofloxacina uma fluorquinolona, seguidos pela ampicilina e sulfametoxazol + trimetoprim, ambos com 70\% (7/10) de resistência, tetraciclina $60 \%$ (6/10), cefazolina e cefotaxima 50\% (5/10), Figura 2.

Foi possível observar que não houve diferença significativa no perfil de resistência a antimicrobianos nos isolados com seleção ou sem previa seleção com ciprofloxacina, sendo a Sulfametoxazol + trimetoprim e a ampicilina os antimicrobianos com maior resistência. Todavia houve diferença na quantidade de isolados com um maior perfil de sensibilidade frente aos antimicrobianos. 
Figura 1. Apresentação do perfil de sensibilidade dos isolados de Escherichia coli isolada de frangos de corte e Alphitobius diaperinus no período pré abate.

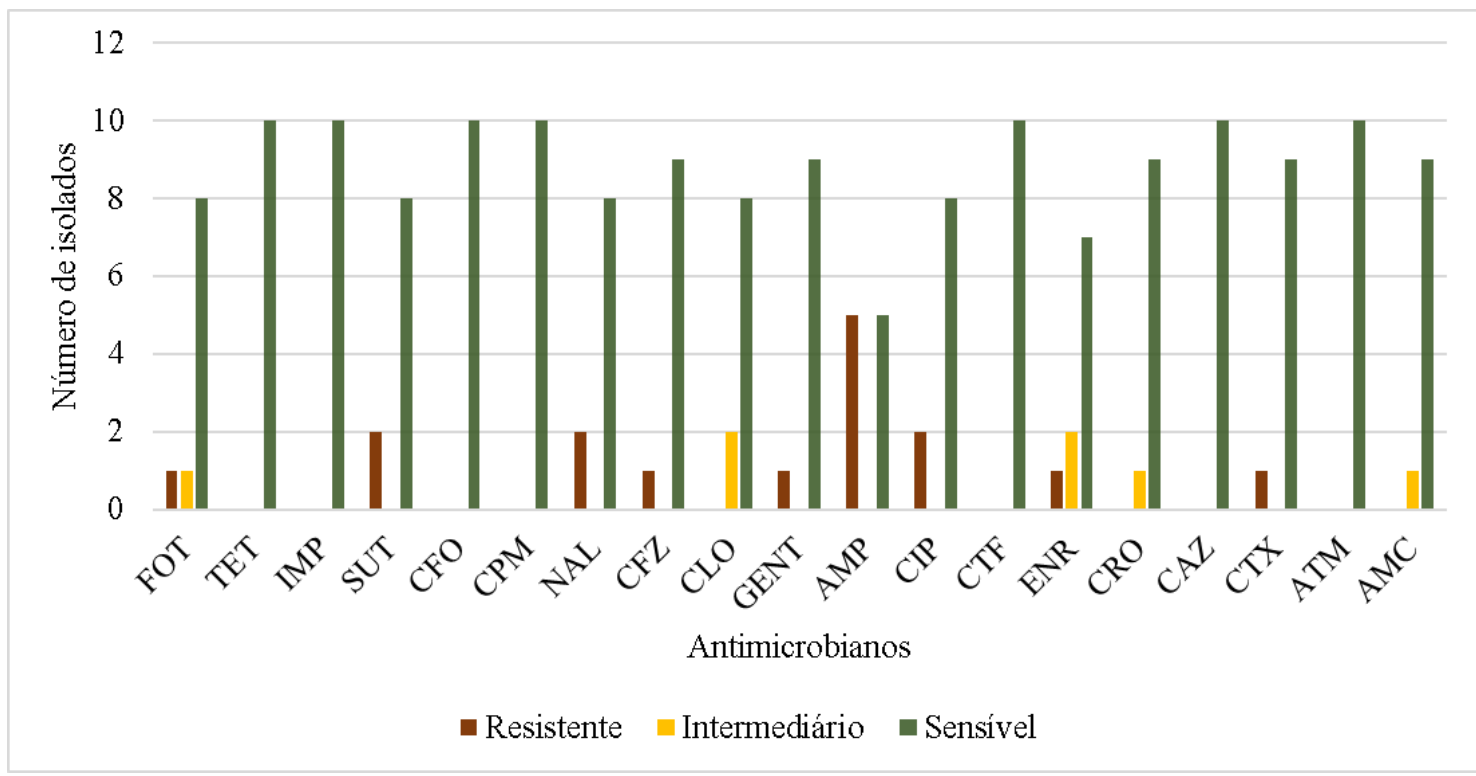

*Amoxacilina - ácido Clavulânico (AMC), Aztreonam (ATM), Cefotaxima (CTX), Ceftadizima (CAZ), Ceftriaxona (CRO), Enrofloxacina (EN), ceftiofur (CTF), Ciprofloxacina (CIP), Ampicilina (AMP), Gentamicina (GEN), Cloranfenicol (CLO), cefazolina (CFZ), Ácido nalidíxico (NAL), Cefepime (CPM), Cefoxitina (CFO), Sulfametoxazol + trimetoprim (SUT), Imipenem (IMP), Tetraciclina (TET), Fosfomicina (FOS).

Fonte: Própria (2020).

Figura 2. Perfil de sensibilidade de E.coli proveniente de suabe cloacal de frangos de corte com isolamento, utilizando MacConkey (8ug/mL cipro).

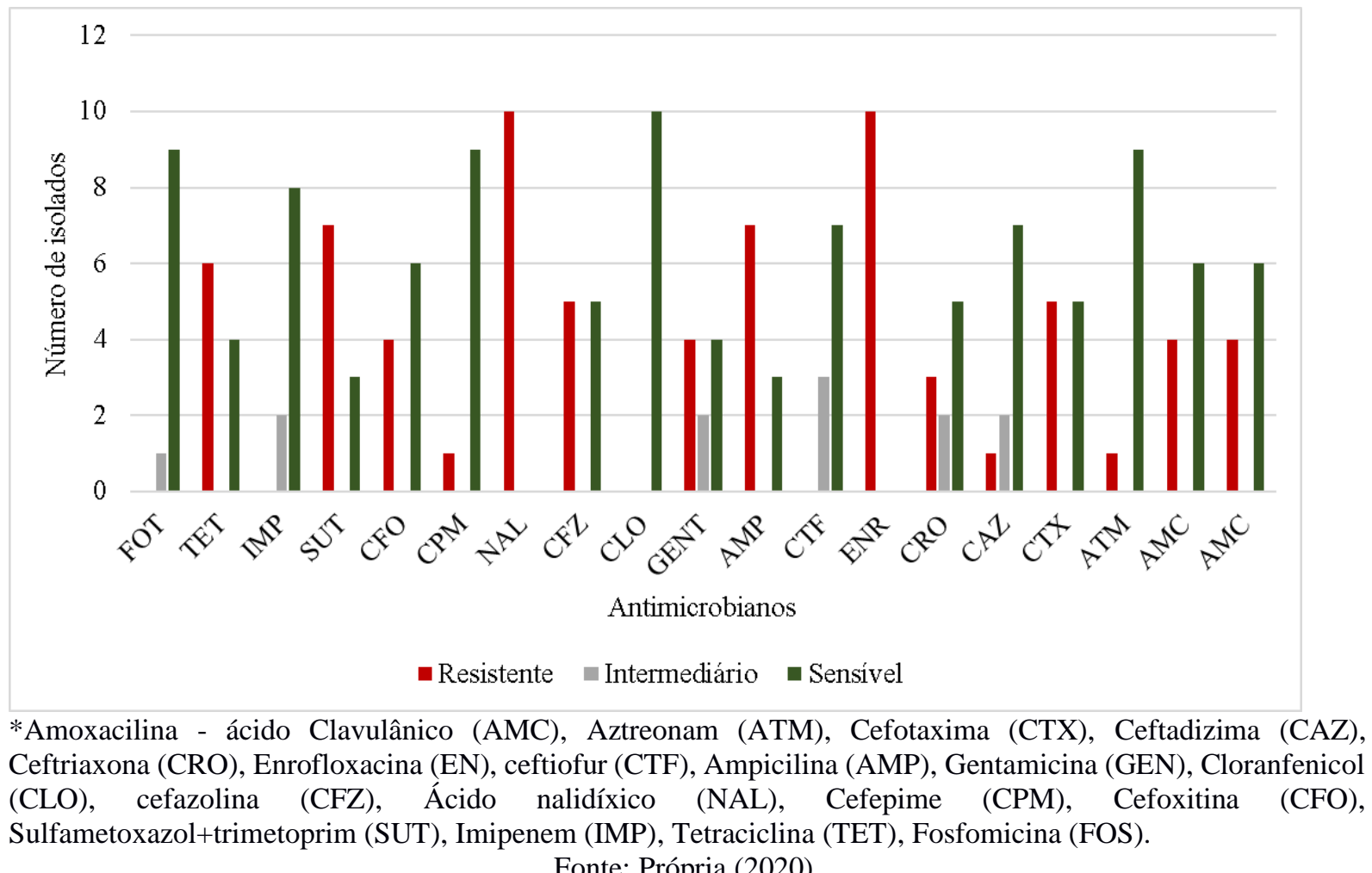
Fonte: Própria (2020).

Foi detectado 100\% (10/10) de perfil MDR em E. coli proveniente de suabe de cloaca 
de frangos com previa seleção (MC 8ug/mL) enquanto 30\% (3/10) dos isolados foram MDR quando não foi feito uso de pré seleção com antimicrobianos.

Aslantaş (2020), na Turquia, encontrou alta resistência ao ácido nalidíxico (92,9\%), ciprofloxacina (76\%), sulfametoxazol-trimetoprim $(78,6 \%)$ e tetraciclina $(73,4 \%)$ em E. coli isolada de frangos de corte Seo, Shim e Lee (2019) trabalharam com 591 isolados de E. coli, e 65 foram resistentes a cefalosporinas de terceira geração, destes 78,5\% apresentaram MDR e $27,7 \%$ foram ESBL positivos.

Foram isoladas quatros cepas de E. coli de Alphitobius diaperinus. Estes insetos podem permanecer na cama do aviário mesmo após a fermentação e podem ficar alojados na nas instalações, fissuras de pisos concretados, alimentando-se de matéria orgânica, fezes e aves mortas, colaborando com a permanência de microrganismos resistentes no ambiente.

E. coli $(\mathrm{n}=2)$ isolada em ágar MacConkey 1, originária de cascudinhos, apresentaram $100 \%(2 / 2)$ de resistência frente a tetraciclina e ácido nalidíxico, seguidos por $50 \%$ (1/2) a fosfomicina, ciprofloxacina e enrofloxacina. A maior sensibilidade foi observada à imipenem, sulfazotrim, cefoxitina, cefepime, cefazolina, cloranfenicol, gentamicina, ampicilina, ceftiofur, cefotaxima, ceftriaxona, aztreonam, ceftazidima e amoxacilina - ácido clavulânico, Tabela 2.

Já E. coli $(\mathrm{n}=2)$ isolada em MacConkey 2, foi observado 100\% (2/2) de resistência a enrofloxacina e ácido nalidíxico, seguidos pela fosfomicina, tetraciclina, cefoxitina, cefepime, cefazolina, gentamicina, ampicilina, ceftriaxona, cefotaxima, amoxacilina- ácido clavulânico, com 50\% (1/2) de resistência. E 100\% (2/2) de sensibilidade a imipenem, sulfazotrim, cloranfenicol, ceftazidima, aztreonam.

Dos isolados de Alphitobius diaperinus somente um de origem sem previa seleção com ciprofloxacina não foi MDR, todos os outros apresentaram-se multirresistentes a drogas.

E pode-se observar que os antimicrobianos com maior resistência foram a tetraciclina, a enrofloxacina, ácido nalidíxico e a fosfomicina, quando comparando-se os meios. Resistência a importantes antimicrobianos foi encontrada em cascudinhos, o que demonstra a necessidade do controle e de medidas de controle para esse coleóptero (JAPP; BICHO; SILVA, 2010).

\section{Teste fenotípico $\beta$-lactamase de espectro expandido}

Com relação a produção de $\beta$-lactamase de espectro expandido, nenhum dos isolados oriundos MC apresentou a enzima. Enquanto dos isolados em MC-CP 50\% (5/10) apresentaram a enzima produtora de ESBL, Tabela 3. 
Tabela 2. Perfil de suscetibilidade a antimicrobianos de E. coli isoladas de Alphitobius diaperinus em ágar MacConkey (MC) e Ágar MacConkey suplementado com Ciprofloxacina (8ug/mL) (MC-CP).

\begin{tabular}{|c|c|c|c|c|c|c|c|}
\hline \multirow{2}{*}{\multicolumn{2}{|c|}{ Meio para seleção/ Antimicrobianos }} & \multicolumn{2}{|c|}{ Resistente } & \multicolumn{2}{|c|}{ Intermediário } & \multicolumn{2}{|c|}{ Sensível } \\
\hline & & MC & $\mathbf{C P}$ & MC & $\mathbf{C P}$ & MC & $\mathbf{C P}$ \\
\hline \multirow{19}{*}{$\begin{array}{l}0 \\
0 \\
0 \\
\frac{0}{0} \\
0 \\
0 \\
0 \\
0 \\
0 \\
0 \\
\Xi \\
\vdots \\
Z\end{array}$} & FOT & 1 & 1 & 0 & 0 & 1 & 1 \\
\hline & TET & 2 & 1 & 0 & 0 & 0 & 1 \\
\hline & IMP & 0 & 0 & 0 & 0 & 2 & 2 \\
\hline & SUT & 0 & 0 & 0 & 0 & 2 & 2 \\
\hline & $\mathrm{CFO}$ & 0 & 1 & 0 & 0 & 2 & 1 \\
\hline & CPM & 0 & 1 & 0 & 0 & 2 & 1 \\
\hline & NAL & 2 & 2 & 0 & 0 & 0 & 0 \\
\hline & CFZ & 0 & 1 & 0 & 0 & 2 & 1 \\
\hline & CLO & 0 & 0 & 0 & 0 & 2 & 2 \\
\hline & GENT & 0 & 1 & 0 & 0 & 2 & 1 \\
\hline & AMP & 0 & 1 & 0 & 0 & 2 & 1 \\
\hline & CIP & 1 & 2 & 0 & 0 & 1 & 0 \\
\hline & CTF & 0 & 0 & 0 & 1 & 2 & 1 \\
\hline & ENR & 1 & 2 & 1 & 0 & 0 & 0 \\
\hline & CRO & 0 & 1 & 0 & 0 & 2 & 1 \\
\hline & CAZ & 0 & 0 & 0 & 0 & 2 & 2 \\
\hline & CTX & 0 & 1 & 0 & 0 & 2 & 1 \\
\hline & ATM & 0 & 0 & 0 & 0 & 2 & 2 \\
\hline & $\mathrm{AMC}$ & 0 & 1 & 0 & 0 & 2 & 1 \\
\hline
\end{tabular}

*Amoxacilina - ácido Clavulânico (AMC), Aztreonam (ATM), Cefotaxima (CTX), Ceftadizima (CAZ), Ceftriaxona (CRO), Enrofloxacina (EN), ceftiofur (CTF), Ciprofloxacina (CIP), Ampicilina (AMP), Gentamicina (GEN), Cloranfenicol (CLO), cefazolina (CFZ), Ácido nalidíxico (NAL), Cefepime (CPM), Cefoxitina (CFO), Sulfametoxazol+trimetoprim (SUT), Imipenem (IMP), Tetraciclina (TET), Fosfomicina (FOS). **MC $=$ ágar MacConkey sem a suplementação com antimicrobianos; $\mathrm{CP}=$ ágar MacConkey acrescido de ciprofloxacina.

Fonte: Própria (2020).

Tabela 3. Número de E. coli isoladas de suabe cloacal de frangos de corte utilizando ágar MacConkey e MacConkey + CIPRO (8ug/mL) quanto a produção da enzima ESBL.

Meios de cultura/ESBL

ESBL Positivo

ESBL Negativo

\begin{tabular}{lcc}
\hline MacConkey & 0 & 10 \\
MacConkey + Ciprofloxacina $(8 \mathrm{ug} / \mathrm{mL})$ & 5 & 5 \\
\hline
\end{tabular}

*ESBL- $\beta$-lactamase de espectro expandido.

Fonte: Própria (2020).

É importante destacar que a ciprofloxacina pertence a classe das quinolonas, enquanto para a avaliação fenotípica de ESBL, são necessários cefalosporinas de $3^{\circ}, 4^{\circ}$ ou $5^{\circ}$ geração e/ ou um monobactâmico.

Estudo realizado por Matsumura et al. (2015) utilizando E. coli isolada de hospitais do Japão, demonstrou correlação entre ciprofloxacina e ESBL, sendo 396 amostras resistentes a 
ciprofloxacina de 1079 isolados de Escherichia coli produtores de ESBL, indicando que 37\% das amostras ESBL positivo eram resistentes a ciprofloxacina.

Falgenhauer et al., (2019) obtiveram 140 amostras fecais de frangos de corte de oito granjas na África, dos quais 41 (29\%) foram ESBL positivos. E eles observaram que existe uma grande diferença na prevalência de ESBL, que pode variar entre 0 a $85 \%$.

Aslantaş (2020) coletou 430 amostras de suabe cloacal de frangos de corte e destas 154 $(35,8 \%)$ foram positivas para E. coli produtora de ESBL / pAmpC.

Awoh et al. (2020) coletaram 111 amostras de fezes de aves, de 52 núcleos distintos. Destas foram isoladas 22 isolados E. coli, aproximadamente $31 \%$ foram ESBL positivos, Nigéria.

Os dados corroboram com os encontrados, nenhum dos isolados de E. coli sem previa seleção apresentaram ESBL positivo, com relação a correlação de resistência a ciprofloxacina e ESBL, com uma previa seleção $50 \%$ dos isolados foram ESBL positivo. Somente dois isolados de MC apresentaram resistência a ciprofloxacina.

\section{Conclusões}

Conclui-se que existe um alto percentual Escherichia coli isoladas da microbiota intestinal de frangos de corte no período pré abate, resistente a sulfametoxazol + trimetoprim, enrofloxacina e a ampicilina, independentemente do tipo de meio de cultura empregado. Os isolados de Escherichia coli, de Alphitobius diaperinus, apresentam alto perfil de resistência, principalmente a tetraciclina, enrofloxacina e fosfomicina. Comparando ambos os meios de cultura utilizados, os isolados em meio Macconkey suplementado com ciprofloxacina apresentaram perfil de resistência a um número maior de antimicrobianos, assim como um maior número de isolados multirresistentes a drogas e produtores de $\beta$-lactamase de espectro estendido.

\section{Referências}

ABPA. Relatório Anual. ABPA- Associação Brasileira de Proteína Animal. 2019.

ASLANTAŞ, Ö.. High occurrence of CMY-2-type beta-lactamase-producing Escherichia coli among broiler flocks in Turkey. Tropical Animal Health and Production, v. 52(4), p. 16811689, 2020.

AWORH, M. K., KWAGA, J., OKOLOCHA, E., HARDEN, L., HULL, D., HENDRIKSEN, R. S., \& THAKUR, S..Extended-spectrum B-lactamase-producing Escherichia coli among humans, chickens and poultry environments in Abuja, Nigeria. One Health Outlook, v. 2(1), p. 8. 2020. 
BIDÊ, P., BONARCORSI, S., \& BINGEN, E. [Virulence factors and pathophysiology of extraintestinal pathogenic Escherichia coli]. Archives De Pediatrie: Organe Officiel De La Societe Francaise De Pediatrie, v. 19(3), p. 80-92, 2012.

BrCAST. Brazilian Committee on Antimicrobial Susceptibility Testing (BrCAST). Versão 6.0, 2017.

CDC. Center for Disease Control and Prevention. Antibiotic/ Antimicrobial Resistance (AR/ AMR). U.s. Department of Health \& Human Services. Disponivel em: < https://www.cdc.gov/drugresistance/index.html >, Acesso em: Junho, 2020.

CHERNAKI-LEFFER, A. M., BIESDORF, S. M., ALMEIDA, L. M., LEFFER, E. V. B., \& VIGNE, F.. Isolamento de enterobactérias em Alphitobius diaperinus e na cama de aviários no oeste do estado do Paraná, Brasil. Brazilian Journal of Poultry Science, v. 4(3), p. 243-247, 2002.

CLSI. Clinical and Laboratory Standards Institute (CLSI). Performance Standards for Antimicrobial Susceptibility Testing. 28th ed. CLSI supplement M100, Wayne, Pennsylvania 19087 USA, 2018.

CLSI. Clinical and Laboratory Standards Institute. Suggested Grouping of US-FDA Approved Antimicrobial Agents That Should Be Considered for Routine Testing and Reporting on Nonfastidious Organisms by Clinical Laboratories. 29ed. CLSI guideline M100-S29. Wayne, PA: Clinical and Laboratory Institute, 2019.

CYOIA, P. S., KOGA, V. L., NISHIO, E. K., HOULE, S., DOZOIS, C. M., DE BRITO, K., DE BRITO, B. G., NAKAZATO, G., \& KOBAYASHI, R.. Distribution of ExPEC Virulence Factors, blaCTX-M, fosA3, and mcr-1 in Escherichia coli Isolated From Commercialized Chicken Carcasses. Frontiers in microbiology, v. 9, p. 3254, 2019.

DAVIES, R.h. \& WRAY, C.. Contribution of the lesser mealworm beetle (Alphitobius diaperinus) to carriage of Salmonella Enteritidis in poultry. The Veterinary Record, v. 137(16), p. 407-408, 1995.

FALGENHAUER, L., IMIRZALIOGLU, C., OPPONG, K., AKENTEN, C. W., HOGAN, B., KRUMKAMP, R., POPPERT, S., LEVERMANN, V., SCHWENGERS, O., SARPONG, N., OWUSU-DABO, E., MAY, J., \& EIBACH, D.. Detection and Characterization of ESBLProducing Escherichia coli From Humans and Poultry in Ghana. Frontiers in Microbiology, v. 9, p. 33582019.

FU, Y., DEIORIO-HAGGAR, K., ANTHONY, J., \& MEYER, M. M.. Most RNAs regulating ribosomal protein biosynthesis in Escherichia coli are narrowly distributed to Gammaproteobacteria. Nucleic Acids Research, v. 41(6), p. 3491-3503, 2013.

GIGUÈRE, S.; PRESCOTT, J. F.; BAGGOT, J. D.; WALKER, R. D.; DOWLING, P. M.. Terapia Antimicrobiana em Medicina Veterinária. 4 ed, São Paulo:Ed. Roca, 2010. 683p

IBGE.

CensoAgro, 2017. https://censoagro2017.ibge.gov.br/templates/censo_agro/resultadosagro/pecuaria.html. 
JAPP, A. K., BICHO, C. DE L., \& SILVA, A. V. F. DA.. Importância e medidas de controle para Alphitobius diaperinus em aviários. Ciência Rural, v. 40(7), p. 1668-1673, 2010.

JOHNSON, T. J., LOGUE, C. M., JOHNSON, J. R., KUSKOWSKI, M. A., SHERWOOD, J. S., BARNES, H. J., DEBROY, C., WANNEMUEHLER, Y. M., OBATA-YASUOKA, M., SPANJAARD, L., \& NOLAN, L. K.. Associations between multidrug resistance, plasmid content, and virulence potential among extraintestinal pathogenic and comensal Escherichia coli from humans and poultry. Foodborne Pathogens and Disease, v. 9(1), p. 37-46, 2012.

KAPER, J. B., NATARO, J. P., \& MOBLEY, H. L. T.. Pathogenic Escherichia coli. Nature Reviews Microbiology, v. 2(2), n. 123-140, 2004.

KUPCZIK, F.; VIALLE, L.R.G.; NOBRE L.O.; VIEIRA, L.A.; FERNANDES, A.E.. Influência da ciprofloxacina na consolidação óssea de fraturas de fêmur em ratos. Acta Ortopédica Brasileira. v.17(4), p. 228-231, 2009.

MAGIORAKOS, A. P., SRINIVASAN, A., CAREY, R. B., CARMELI, Y., FALAGAS, M. E., GISKE, C. G., HARBARTH, S., HINDLER, J. F., KAHLMETER, G., OLSSONLILJEQUIST, B., PATERSON, D. L., RICE, L. B., STELLING, J., STRUELENS, M. J., VATOPOULOS, A., WEBER, J. T., \& MONNET, D. L.. Multidrug-resistant, extensively drugresistant and pandrug-resistant bacteria: an international expert proposal for interim standard definitions for acquired resistance. Clinical microbiology and infection: the official publication of the European Society of Clinical Microbiology and Infectious Diseases, v.18(3), p.268-281, 2012.

MATSUMURA, Y., JOHNSON, J. R., YAMAMOTO, M., NAGAO, M., TANAKA, M., TAKAKURA, S., ICHIYAMA, S., KYOTO-SHIGA CLINICAL MICROBIOLOGY STUDY GROUP, \& KYOTO-SHIGA CLINICAL MICROBIOLOGY STUDY GROUP.. CTX-M-27and CTX-M-14-producing, ciprofloxacin-resistant Escherichia coli of the H30 subclonal group within ST131 drive a Japanese regional ESBL epidemic. Journal of Antimicrobial Chemotherapy, v. 70(6), p. 1639-1649, 2015.

MELLATA, M.. Human and avian extraintestinal pathogenic Escherichia coli: infections, zoonotic risks, and antibiotic resistance trends. Foodborne Pathogens and Disease, v. 10(11), p. $916-932,2013$.

NEU HC.. The crisis in antibiotic resistance. Science, v.257, p.1064-1073, 1992.

OECD/FAO, OECD-FAO Agricultural Outlook 2019-2028, OECD Publishing, Paris, 2019. https://doi.org/10.1787/agr_outlook-2019-en.

OIKARAINEN, P. E., POHJOLA, L. K., PIETOLA, E. S., \& HEIKINHEIMO, A.. Direct vertical transmission of ESBL/pAmpC-producing Escherichia coli limited in poultry production pyramid. Veterinary Microbiology, v. 231, p. 100-106, 2019.

O'NEILL.. Antimicrobial Resistance: Tackling a Crisis for the Health and Wealth of Nations. Review on Antimicrobial Resistance, 2014. Acessado em 20.08.2019. http://amrreview.org/sites/default/files/AMR\%20Review\%20Paper\%20-

20Tackling $\% 20 \mathrm{a} \% 20$ crisis $\% 20$ for $\% 20$ the $\% 20$ health $\% 20$ and $\% 20$ wealth $\% 20$ of $\% 20$ nations_1. pdf. 
PESCIAROLI, M., MAGISTRALI, C. F., FILIPPINI, G., EPIFANIO, E. M., LOVITO, C., MARCHI, L., MARESCA, C., MASSACCI, F. R., ORSINI, S., SCOCCIA, E., TOFANI, S., \& PEZZOTTI, G.. Antibiotic-resistant commensal Escherichia coli are less frequently isolated from poultry raised using non-conventional management systems than from conventional broiler. International Journal of Food Microbiology, v. 314, p. 108391, 2020.

PITOUT, J. D. D.. Extraintestinal pathogenic Escherichia coli: a combination of virulence with antibiotic resistance. Frontiers in Microbiology, v. 3, n. 9, pp. 1-7, 2012.

SEO, K. W., SHIM, J. B., \& LEE, Y. J.. Emergence of CMY-2-Producing Escherichia coli in Korean Layer Parent Stock. Microbial Drug Resistance (Larchmont, N.Y.), v. 25(3), p. 462468, 2019.

THANNER, S.; DRISSNER, D.; WALSH F.. Antimicrobial Resistance in Agriculture. MBio, American Society for Microbiology. v. 7(2), 2016.

THEOBALD, S., ETTER, E. M. C., GERBER, D., \& ABOLNIK, C.. Antimicrobial Resistance Trends in Escherichia coli in South African Poultry: 2009-2015. Foodborne Pathogens and Disease, v.16(9), p. 652-660, 2019.

VAN IMMERSEEL, F., LYHS U., PEDERSEN K., PRESCOTT J.F.. Recent breakthroughs have unveiled the many knowledge gaps in Clostridium perfringens associated necrotic enteritis in chickens: the first International Conference on Necrotic Enteritis in Poultry. Avian Pathology, v. 45, n. 3, p.269-270, 2016.

VITTORI, J., SCHOCKEN-ITURRINO, R. P., TROVÓ, K. P., RIBEIRO, C. A. M., BARBOSA, G. G., DE SOUZA, L. M., \& PIGATTO, C. P. ([s.d.]).. Alphitobius diaperinus como veiculador de Clostridium perfringens em granjas avícolas do interior paulista - Brasil.

Ciência Rural. v. 37(3), 2007.

WILLIAMS, K. P., GILLESPIE, J. J., SOBRAL, B. W. S., NORDBERG, E. K., SNYDER, E. E., SHALLOM, J. M., \& DICKERMAN, A. W. .Phylogeny of Gammaproteobacteria. Journal of Bacteriology, v. 192(9), p. 2305-2314, 2019.

WITTE W.. Medical consequences of antibiotic use in agriculture. Science, v. 279, p. 996-997. 1998.

WHO, Boletim da Organização Mundial da Saúde 2020; 98: 442-442A. doi: http://dx.doi.org/10.2471/BLT.20.268573. Disponível em

https://www.who.int/bulletin/volumes/98/7/20-268573/en/ > Acesso em : Agosto, 2020.

YASSIN, A. K., GONG, J., KELLY, P., LU, G., GUARDABASSI, L., WEI, L., HAN, X., QIU, H., PRICE, S., CHENG, D., \& WANG, C.. Antimicrobial resistance in clinical Escherichia coli isolates from poultry and livestock, China. PLoS ONE, v.12(9), p. 1-8, 2017. 